\title{
Effect of Thermal Buoyancy on Vortex-Shedding and Aerodynamic Characteristics for Fluid Flow Past an Inclined Square Cylinder
}

\author{
Md. Reyaz Arif*, Nadeem Hasan
}

Computational Fluid Dynamics Laboratory, Mechanical Engineering Department, Zakir Husain College of Engineering and Technology, Aligarh Muslim University, Aligarh 202002, India

Corresponding Author Email: reyazarif@zhcet.ac.in

https://doi.org/10.18280/ijht.380223

Received: 23 April 2019

Accepted: 10 February 2020

\section{Keywords:}

vortex shedding, convection, inclined square cylinder, buoyancy, critical, Richardson number

\begin{abstract}
The present work aims to numerically investigate the upward flow of air past an inclined square cylinder with an incidence angle equal to $45^{\circ}$ in the mixed convection regime at a fixed Reynolds number $\mathrm{Re}=100$. The governing equations are modelled by considering Boussinesq approximation. The critical Richardson number $\left(\mathrm{Ri}_{\mathrm{c}}\right)$ for the suppression of vortex shedding (VS) is determined. By observing the instantaneous streamline patterns and the coefficient of lift plot with time near the critical value, it is found that VS suppression occurs at $\mathrm{Ri}_{\mathrm{c}}=0.78$. Also, the aerodynamic characteristics such as lift and drag coefficient, the heat transfer characteristic, and the Strouhal number are studied by varying $\mathrm{Ri}$ in the range of $[0.2,1]$. The role of thermal buoyancy on the aerodynamic parameters such as drag and lift coefficients, Nusselt number and Strouhal number are also studied in the mixed convection regime. It is found that mean drag coefficient and Nusselt number increases with the increase in Ri, and no lift is developed for any Ri, while the Strouhal number increases with increase in Ri and vanishes at a critical Richardson number. Further, the effect of thermal buoyancy on streamlines, isotherms and iso-vorticity contours are also presented.
\end{abstract}

\section{INTRODUCTION}

The study of vortex shedding (VS) phenomena for the flow over a bluff body (circular and square cylinder) have been a topic of interest due to its application in cooling of electronic devices, heat exchangers, mixing phenomena, design of bridge and cooling towers [1-6]. The onset of VS occurs at some critical Reynolds number, which depends on the geometry of bluff bodies. This VS is sometimes desirable while its suppression is necessary in other cases. VS is favorable in the case of heat and mass transfer for better mixing, while its suppression is needed where there is a chance of failure of structures such as flow past a bridge, chimneys, tall buildings, etc. Therefore, the control of VS past a cylinder is an important aspect of research [7-13]. Several works are available related to mixed convective flows past a square or circular cylinder [14-16]. Sharma and Eswaran [17] studied numerically the VS process for $2 \mathrm{D}$ flow past a heated square cylinder $\left(\alpha=0^{\circ}, \phi=0^{\circ}\right)$ kept at constant temperature for $\operatorname{Re}=100, \operatorname{Pr}=0.7$, and found that VS suppression occurs at $\mathrm{Ri}_{\mathrm{c}}=0.15$. While the vortex shedding phenomena were studied by Bhattacharyya and Mahapatra [18] for the cross-flow of air past a heated square cylinder $\left(\phi=0^{\circ}\right)$ at $\mathrm{Re}=100-1000$. Employing Boussinesq model, they found that no VS suppression occurs for any value of $\mathrm{Ri}$ in the cross-flow configuration. They also studied the variation of aerodynamic parameters with Ri. Hasan and Ali [19] numerically determined the neutral curves, which separates the steady flow from unsteady flow for square $\left(\phi=0^{\circ}\right)$ and circular cylinder in $\mathrm{Ri}-\alpha$ plane at $\mathrm{Re}=60$ and 100 . Also, the detailed explanation of VS suppression mechanism for low heating was proposed. Recently, Hasan and Saeed [20] used a
non-Boussinesq model to study numerically the flow of air past a square cylinder $\left(\phi=0^{\circ}\right)$ in forced convection regime. They studied the combined influence of heating and freestream inclination at three different Reynolds numbers. Chatterjee and Mondal [21] used CFD software FLUENT and performed numerical simulations to study the influence of aiding/opposing buoyancy on the upward flow past a heated/cooled cylinder of square cross-section at $\mathrm{Re}=50-150$. Two different blockage ratios are taken, one corresponding to near un-confined configuration while others correspond to channel confined configuration. They found that for both the confined and unconfined cases the critical Ri increases with $\mathrm{Re}$, while there is increase in $\mathrm{Ri}_{\mathrm{c}}$ with decrease in blockage ratio for all Reynolds number. Sahu et al. [22] numerically studied the influence of Re and Pr on the heat transfer for the cross-flow past an unconfined heated square cylinder for $\mathrm{Re}=60-160, \operatorname{Pr}=0.7-50$, and $\phi=0^{\circ}$. Using two different thermal boundary conditions, namely constant wall temperature condition, and constant heat flux condition, they calculated the overall mean and surface averaged $\mathrm{Nu}$. They found that the overall mean $\mathrm{Nu}$ increases by increasing $\mathrm{Re}$ and Pr. Tanweer et al. [23] numerically investigated the effect of Pr on the mixed convective flow past a square cylinder in cross-flow configuration. Reynolds and Richardson numbers are kept fixed at $R e=100$, and $R i=1.0$, while the $P r$ is varied in the range $[0.02,100]$. They showed that the mean coefficient of lift is negative for low Pr, while it is positive at very high Pr. Elsherbiny et al. [24] experimentally studied the effect of free convection and angle of attack on the heat transfer for flow past an isothermal square cylinder at Re varied from 48 to 670 .

The literature survey shows that most of the studies on the 
flow past a cylinder of square cross-section in mixed convection regime have been carried out for inclination, $\phi=$ $0^{\circ}$. Some of the works are available for the flow over an unheated inclined square cylinder at low Re [25-27] While very few works are available for forced and mixed convective flows past an inclined square cylinder. Ranjan et al. [28] performed numerical simulation for cross-flow to study the flow of air past a heated inclined square cylinder in forced convection regime. They studied the variation of Strouhal number, aerodynamic forces, moment and Nusselt number with cylinder inclination in the range, $\phi=\left[0,45^{\circ}\right]$ at Re varying in range, $\mathrm{Re}=[60,150]$. Dulhani et al. [29] performed numerical experiments to study the cross-flow past a square cylinder at incidence in mixed convection regime at $\mathrm{Re}=100$. They performed detailed study of the aerodynamics and heat transfer parameters at $\mathrm{Ri}$ and $\phi$ in the range of $[-1,1]$ and $[0$, $45^{\circ}$ ], respectively. Kakade et al. [30] experimentally studied the combined effects of square cylinder inclination and buoyancy at fixed free-stream orientation $\left(\alpha=0^{\circ}\right)$ at $\mathrm{Re}=56$, 87 and 100. They found that with the increase in cylinder inclination the critical Richardson number increases. Arif and Hasan [31] studied vortex shedding suppression characteristics for mixed convective flow past a square cylinder in a large-scale heating regime. Employing nonBoussinesq model by varying cylinder inclination and freestream inclination in the range $\left[0,45^{\circ}\right]$, and $\left[0,90^{\circ}\right]$ respectively, they developed the neutral curves separating steady and unsteady flow regimes.

Thus, from the above literature survey, it is found that the study of vortex shedding suppression phenomena and the aerodynamic and heat transfer study for the upward flow (aiding buoyancy) past a square cylinder inclined at $\phi=45^{\circ}$ in mixed convection regime (small-scale heating) is not yet explored numerically. Thus, the present work aims to study the effect of thermal buoyancy on the upward flow of air past a cylinder of square cross-section inclined at an angle $\phi=45^{\circ}$. The critical Ri for the suppression of VS is determined within the framework of Boussinesq approximation. Also, the effect of thermal buoyancy on the aerodynamics parameters and heat transfer characteristics is studied by varying $\mathrm{Ri}$ in the range $0.2 \leq \mathrm{Ri} \leq 1.0$. Furthermore, the effect of thermal buoyancy on the various flow patterns such as streamline, vorticity contours and isotherms are presented. The various dimensionless flow parameters are $\operatorname{Re}=100, \operatorname{Pr}=0.71, \alpha=0^{\circ}, \phi=45^{\circ}$ and $0.2 \leq \mathrm{Ri} \leq 1.0$

\section{MATHEMATICAL MODELLING}

The formulation of the governing equations is done by considering the flows to be two-dimensional, unsteady, laminar and incompressible. The variation of coefficient of viscosity, thermal conductivity, and specific heat with temperature are neglected, which means the constant properties model is used. The density variation is neglected everywhere, except when the density is coupled with body forces such as gravity. This implies that the Boussinesq approximation is made, i.e. density is approximated by a relation $\rho=\rho_{\infty}\left[1-\beta\left(T-\mathrm{T}_{\infty}\right)\right]$, where $\beta$ is thermal expansion coefficient. The temperature difference between the freestream and the cylinder surface temperature is maintained small for the justification of constant transport and the specific heat properties consideration. The governing equations with various non-dimensional parameters and boundary conditions are described in the forthcoming subsection.

\subsection{Governing equations}

In the present work, small-scale heating is considered, hence Boussinesq approximation is used for formulation. The continuity, $\mathrm{X}$ and $\mathrm{Y}$ components of momentum and energy equations in dimensionless form are respectively expressed as;

$$
\begin{gathered}
\frac{\partial \mathrm{U}}{\partial \mathrm{X}}+\frac{\partial \mathrm{V}}{\partial \mathrm{Y}}=0 \\
\frac{\partial \mathrm{U}}{\partial \tau}+\frac{\partial(\mathrm{UU})}{\partial \mathrm{X}}+\frac{\partial(\mathrm{VU})}{\partial \mathrm{Y}}=-\frac{\partial \mathrm{P}}{\partial \mathrm{X}}+\frac{1}{\operatorname{Re}\left(\frac{\partial^{2} \mathrm{U}}{\partial \mathrm{X}^{2}}+\frac{\partial^{2} \mathrm{U}}{\partial \mathrm{Y}^{2}}\right)} \\
\frac{\partial \mathrm{V}}{\partial \tau}+\frac{\partial(\mathrm{UV})}{\partial \mathrm{X}}+\frac{\partial(\mathrm{VV})}{\partial \mathrm{Y}}=-\frac{\partial \mathrm{P}}{\partial \mathrm{Y}}+\frac{1}{\operatorname{Re}}\left(\frac{\partial^{2} \mathrm{~V}}{\partial \mathrm{X}^{2}}+\frac{\partial^{2} \mathrm{~V}}{\partial \mathrm{Y}^{2}}\right)+\operatorname{Ri} \theta \\
\frac{\partial \theta}{\partial \tau}+\frac{\partial(\mathrm{U} \theta)}{\partial \mathrm{X}}+\frac{\partial(\mathrm{V} \theta)}{\partial \mathrm{Y}}=\frac{1}{\operatorname{RePr}}\left(\frac{\partial^{2} \theta}{\partial \mathrm{X}^{2}}+\frac{\partial^{2} \theta}{\partial \mathrm{Y}^{2}}\right)
\end{gathered}
$$

where, the dimensionless variables are given by,

$$
\mathrm{U}=\frac{\mathrm{u}}{\mathrm{U}_{\infty}}, \mathrm{V}=\frac{\mathrm{v}}{\mathrm{U}_{\infty}}, \tau=\frac{\mathrm{tU}_{\infty}}{\mathrm{d}}, \mathrm{X}=\frac{\mathrm{x}}{\mathrm{d}}, \mathrm{Y}=\frac{\mathrm{y}}{\mathrm{d}}, \mathrm{P}=\frac{\mathrm{p}-\mathrm{p}_{\infty}}{\rho \mathrm{U}_{\infty}^{2}}, \theta=\frac{\mathrm{T}-\mathrm{T}_{\infty}}{\mathrm{T}_{\mathrm{w}}-\mathrm{T}_{\infty}}
$$

$\mathrm{U}_{\infty}, \mathrm{T}_{\infty}$ and $\mathrm{d}$ is considered as free-stream velocity, freestream temperature and edge of the cylinder, respectively. The cylinder surface temperature is denoted by $\mathrm{T}_{\mathrm{w}}$ and is kept slightly higher than $\mathrm{T}_{\infty}$. The value of free-stream temperature is taken as $300 \mathrm{~K}$. The various dimensionless flow parameters used are Reynolds number, Richardson number and Prandtl number respectively defined as,

$$
\mathrm{Re}=\frac{\mathrm{U}_{\infty} \mathrm{d}}{v}, \mathrm{Ri}=\frac{\mathrm{g} \beta\left(\mathrm{T}_{\mathrm{w}}-\mathrm{T}_{\infty}\right) \mathrm{d}}{\mathrm{U}_{\infty}{ }^{2}} \text { and } \operatorname{Pr}=\frac{v}{\alpha},
$$

where, the symbol $v, \beta$ and $\alpha$ represents fluids kinematic viscosity, coefficient of thermal expansion and thermal diffusivity respectively. The non-dimensional output parameters which are used in the text are described below. The lift and the drag coefficients are respectively given by,

$$
C_{L}=\frac{F_{L}}{\frac{1}{2} \rho U_{\infty}^{2} d}, C_{D}=\frac{F_{D}}{\frac{1}{2} \rho U_{\infty}^{2} d}
$$

where, the lift and drag forces acting on the cylinder are denoted by $F_{L}$ and $F_{D}$ respectively.

The non-dimensional form of the heat transfer parameter is Nusselt number, which gives the quantitative information of the heat transfer from the surface of square cylinder to the surrounding fluid. It is expressed by a relation,

$$
\mathrm{Nu}=\frac{\mathrm{Q}}{4 \mathrm{k}\left(\mathrm{T}_{\mathrm{w}}-\mathrm{T}_{\infty}\right)}
$$

The VS frequency in non-dimensional form is known as Strouhal number. It gives the frequency at which the vortices shed in the Von-Karman vortex street in the dimensionless form. It is given by a relation, 


$$
\mathrm{St}=\frac{\mathrm{fd}}{\mathrm{U}_{\infty}}
$$

where, $\mathrm{f}$ is the dimensional form of VS frequency.

\subsection{Boundary conditions}

In the present numerical simulations, the various boundary conditions in non-dimensional form are described below: Inlet:

$$
\mathrm{U}=0, \mathrm{~V}=1, \theta=0
$$

Outlet: Outflow boundary condition i.e.

$$
\frac{\partial \omega}{\partial Y}=0
$$

where, $\omega=\mathrm{U}, \mathrm{V}$ and $\theta$,

Cylinder surfaces:

$$
\mathrm{U}=0, \mathrm{~V}=0, \theta=1
$$

Left and right artificial boundaries:

$$
\frac{\partial \mathrm{V}}{\partial \mathrm{X}}=0, \mathrm{U}=0, \frac{\partial \theta}{\partial \mathrm{X}}=0
$$

The computational domain with physical description of the flow problem is depicted in Figure 1. The distance between the free slip boundary is computational length of the domain and is taken as $\mathrm{L}=80 \mathrm{~d}$. The total height of computation is $\mathrm{H}=\mathrm{H}_{\mathrm{u}}$ $+\mathrm{H}_{\mathrm{d}}=50 \mathrm{~d}$, the upstream height is $\mathrm{H}_{\mathrm{u}}=10 \mathrm{~d}$ while the downstream height is $\mathrm{H}_{\mathrm{d}}=40 \mathrm{~d}$. It is justified to use this type of computational domain as it is consistent with the appropriate domain proposed by Sharma and Eswaran [32] and Yang and $\mathrm{Wu}$ [33] for mixed convective unconfined upward flow of air past a square cylinder at $\mathrm{Re}=100$.

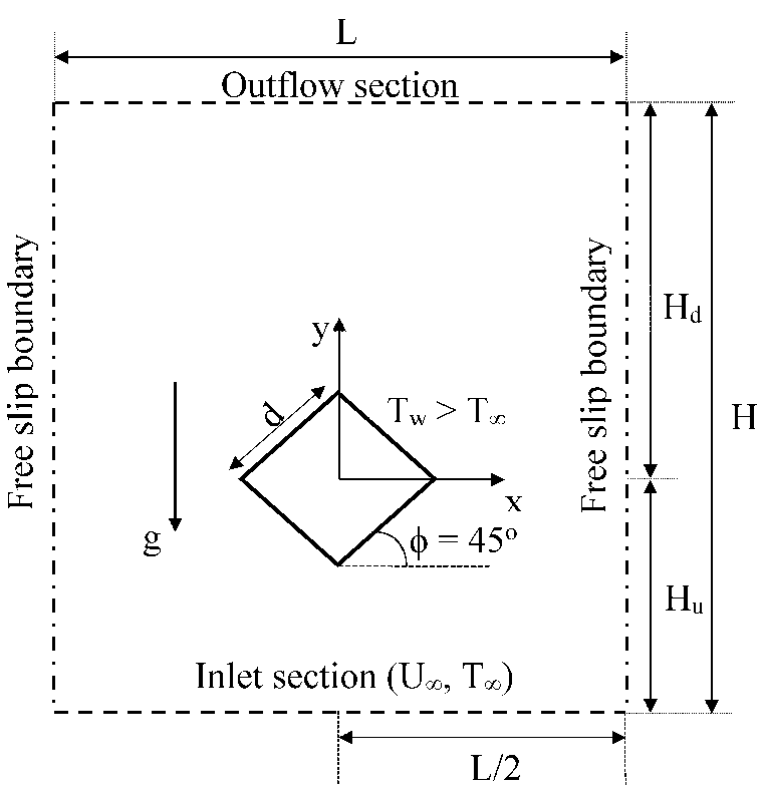

Figure 1. Schematic diagram of flow problem showing computational domain

\section{COMPUTATIONAL DOMAIN}

\subsection{Numerical methods}

The numerical simulations are performed by solving governing equations (Eqns. (1) - (4)) and boundary conditions (Eqns. (10) - (13)) using a finite volume method (FVM) solver commonly known as ANSYS FLUENT 16.0 [34]. The unstructured mesh with clustering of node point in the vicinity of cylinder edge is generated using the ICEM-CFD package. The mesh independence test is performed on three different mesh sizes, and the comparison of the mean drag and mean $\mathrm{Nu}$ is done between these meshes. It is found that the mesh containing node points 18533 , triangular elements 36588 , and minimum grid size 0.01 is the most appropriate mesh and hence is used for all numerical computations. The unstructured mesh with an enlarged view is shown in Figure 2.

The fractional step scheme is used for pressure-velocity coupling and used to solve the continuity and momentum equation and are described below:

The intermediate velocity $\mathrm{u}^{*}$ is calculated by ignoring pressure term

$$
\frac{\mathrm{u}^{*}-\mathrm{u}^{\mathrm{n}}}{\Delta \mathrm{t}}=-\left(\mathrm{u}^{\mathrm{n}} \cdot \nabla\right) \mathrm{u}^{\mathrm{n}}+v \nabla^{2} \mathbf{u}^{\mathrm{n}}
$$

The final solution of the time step $\mathrm{u}^{\mathrm{n}+1}$, intermediate velocity $\mathrm{u}^{*}$ is now can be written as

$$
\mathrm{u}^{(\mathrm{n}+1)}=\mathrm{u}^{*}-\frac{\Delta \mathrm{t}}{\rho} \nabla \mathrm{p}^{(\mathrm{n}+1)}
$$

Rewriting the above equation in form of time step as

$$
\frac{\mathrm{u}^{(\mathrm{n}+1)}-\mathrm{u}^{*}}{\Delta \mathrm{t}}=-\frac{1}{\rho} \nabla \mathrm{p}^{(\mathrm{n}+1)}
$$

where, $\mathrm{n}$ and $(\mathrm{n}+1)$ denote the current and next time step of the solution respectively.

By taking the divergence of continuity condition at $(n+1)$ time level $\nabla \cdot \mathbf{u}^{(\mathrm{n}+1)}=0$ and applying the projector equation (Eq. (14)), the following Poisson equation is obtained,

$$
\nabla^{2} \mathrm{p}^{(\mathrm{n}+1)}=\frac{\rho}{\Delta \mathrm{t}} \nabla \cdot \mathrm{u}^{*}
$$

After solving the Poisson equation using the Gauss-Seidel type algebraic multigrid method for pressure at $(n+1)$ time step, the velocity field can be updated by Eq. (14).

The discretization of convective terms in the momentum and energy equations is done by QUICK (Quadratic Upstream Interpolation for Convective Kinetics scheme) which is second order central difference for a diffusive term, and third order accurate in space and first order in time for a convective term. For the spatial discretization, gradient terms are discretized using least squares cell based, pressure by second order. For time accuracy, a second order implicit scheme is used. The Non-Iterative Time Advancement Scheme (NITA) is used as a solution method. In the NITA scheme, the transient simulation speeds up, as each set of the equation is solved using inner iteration and only one outer iteration per time step. The time-step size of 0.0005 is used for all computations, since the smaller value of time-step do not produce any significant changes in the various output parameters used in present work. 


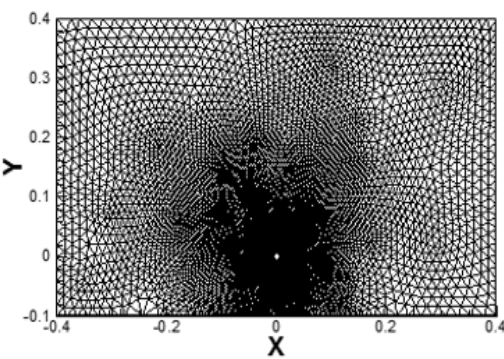

(a)

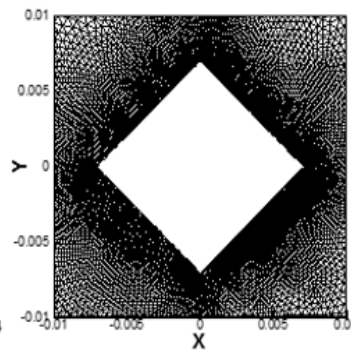

(b)
Figure 2. (a) Unstructured grid; (b) Enlarged view of the grid

\subsection{Validation studies}

In order to ensure the accuracy and reliability of the numerical methods and various other numerical aspects, the validation of the present numerical solver is done with the work of Sohankar et al. [26]. For this, numerical simulation is performed for the flow of air past an unheated square cylinder inclined at an incidence angle of $\phi=45^{\circ}$. Sohankar et al. [26] define $\mathrm{Re}, \mathrm{C}_{\mathrm{D}}, \mathrm{C}_{\mathrm{L}}$ with respect to the cylinder projected width $\mathrm{d}_{\mathrm{p}}=\mathrm{d}(\cos \phi+\sin \phi)$ along the orientation of free-stream, while the cylinder edge is used for the present work. Thus, the Reynolds number $\left(\operatorname{Re}^{*}\right)$, coefficient of lift $\left(\mathrm{C}_{\mathrm{L}}{ }^{*}\right)$, and the Strouhal number $\left(\mathrm{St}^{*}\right)$ used by them and in present work are related by,

$$
\operatorname{Re}=\frac{\operatorname{Re}^{*}}{(\cos \phi+\sin \phi)}, C_{D}{ }^{*}=\frac{C_{D}}{(\cos \phi+\sin \phi)}, \mathrm{St}^{*}=\operatorname{St}(\cos \phi+\sin \phi)
$$

Table 1. Validation of present results with Sohankar et al. [26] at $\operatorname{Re}^{*}=100, \operatorname{Pr}=0.71$ and $\phi=45^{\circ}$

\begin{tabular}{ccc}
\hline Source & CD $_{\mathbf{D}}$ & St \\
\hline Sohankar et al. [26] & 1.721 & 0.179 \\
Present & 1.802 & 0.184
\end{tabular}
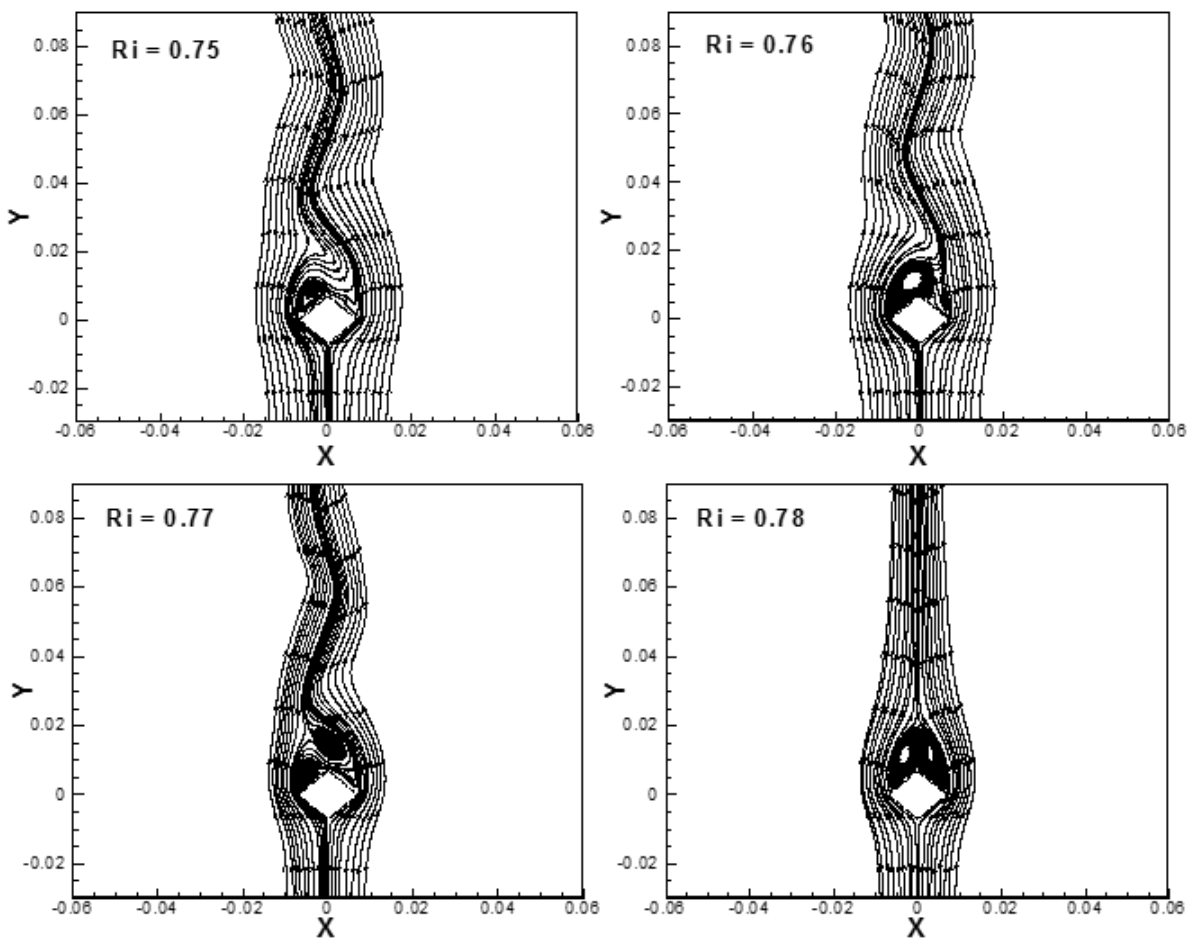

Figure 3. Instantaneous streamline patterns at $\mathrm{Ri}=0.75,0.76,0.77$ and 0.78
The comparison of the coefficient of drag and Strouhal frequency is made with earlier reported work as shown in Table 1, which shows a good agreement.

\section{RESULTS AND DISCUSSIONS}

\subsection{Effect of thermal buoyancy in suppression of VS}

For the upward flow of air past a circular or square cylinder in the Reynolds number range varying from 50 to 150 , the VS phenomena occur and the downstream region is known as Von-Karman vortex street [35]. Thermal buoyancy is one of the active methods to suppress VS. As the heat to the cylinder increases (i.e., increasing Ri), the thermal buoyancy increases and at a certain critical heat input i.e. critical Richardson number, the VS suppression occurs leading to the degeneration of Von-Karman vortex street. The detailed mechanism of VS suppression by buoyancy was given by Hasan and Ali [19]. To determine the VS suppression phenomenon, a series of numerical experiments are performed near critical Ri. In the present work, the simulations are performed in the Ri varying in the range of $0.2 \leq \mathrm{Ri} \leq 1.0$. The instantaneous streamline patterns at $\mathrm{Ri}=0.75,0.76,0.77$ and 0.78 are shown in Figure 3 , while the time histories of lift coefficients are shown in Figure 4. From Figure 3 it is observed that the instantaneous streamline patterns show VS at $\mathrm{Ri}=0.75,0.76$ and 0.77 while at $\mathrm{Ri}=0.78$, the VS is suppressed. In Figure 4 the amplitude of coefficient of lift time series shows a non-zero equilibrium value at $\mathrm{Ri}=0.75,0.76$ and 0.77 . While at $\mathrm{Ri}=0.78$, the amplitude of the coefficient of lift time series decreases and will attain a zero value in the limit of large times. This confirms that the critical value of $\mathrm{Ri}$ for VS suppression is $\mathrm{Ri}_{\mathrm{c}}$ $=0.78$, when the flow variables are $\alpha=0^{\circ}, \phi=45^{\circ}, \operatorname{Re}=100$, and $\operatorname{Pr}=0.71$. Thus, it is found that the increase in thermal buoyancy changes the flow from unsteady regime to steady regime, causing suppression in vortex shedding at a critical value $(\mathrm{Ri}=0.78)$ 

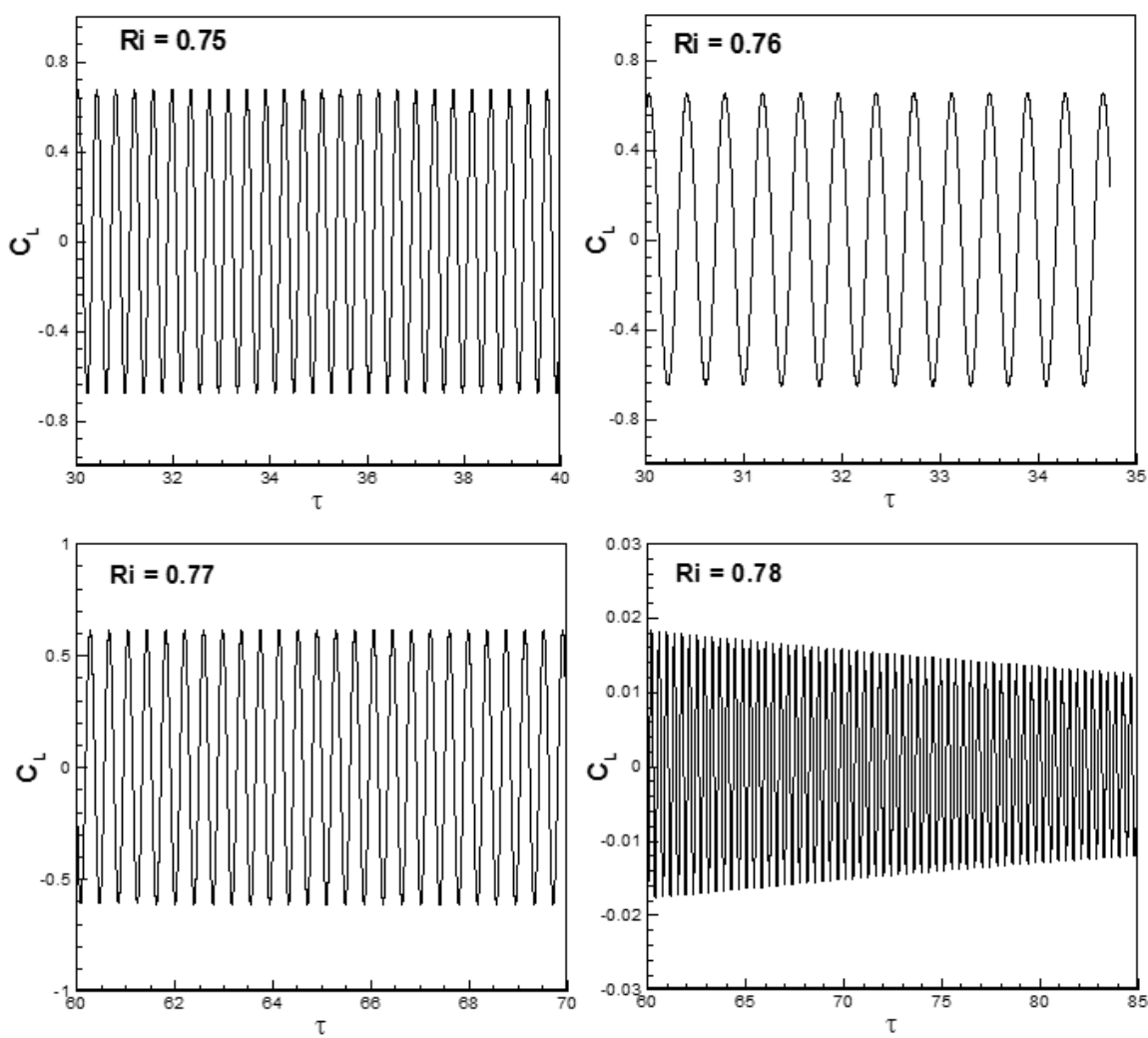

Figure 4. Time history plot of lift coefficient at $\mathrm{Ri}=0.75,0.76,0.77$ and 0.78

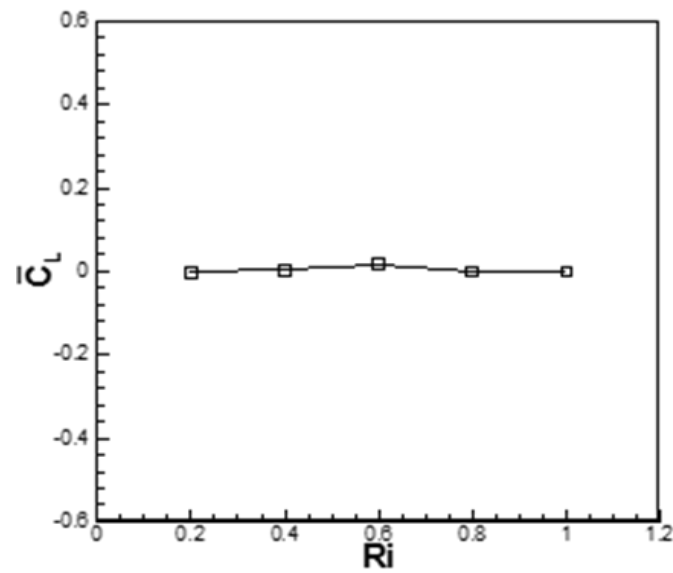

(a)

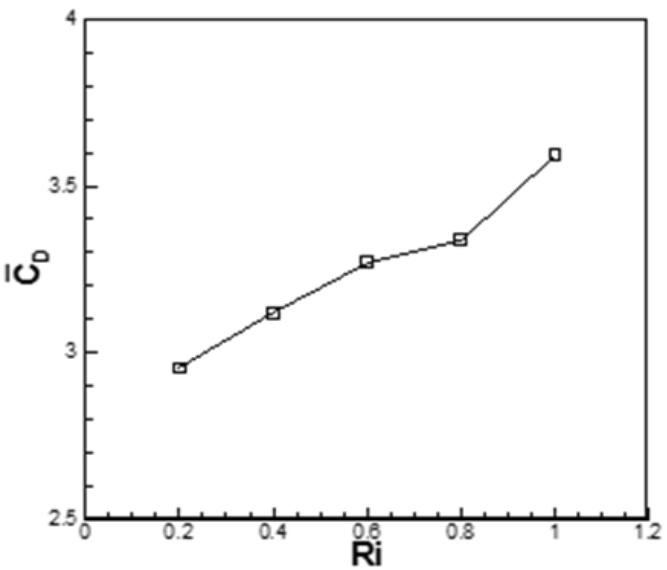

(b)

Figure 5. (a) Variation of mean lift coefficient with Ri (b) Variation of mean drag coefficient with Ri
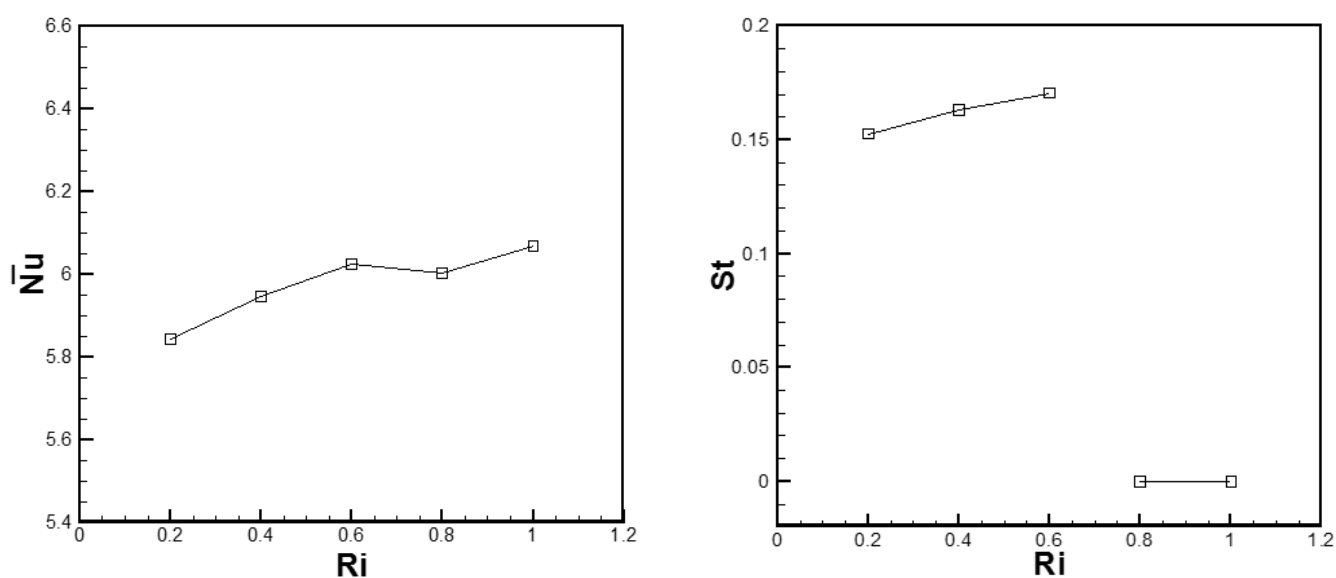

Figure 6. Mean Nusselt number Vs Richardson number

Figure 7. Variation of Strouhal number with Richardson number 

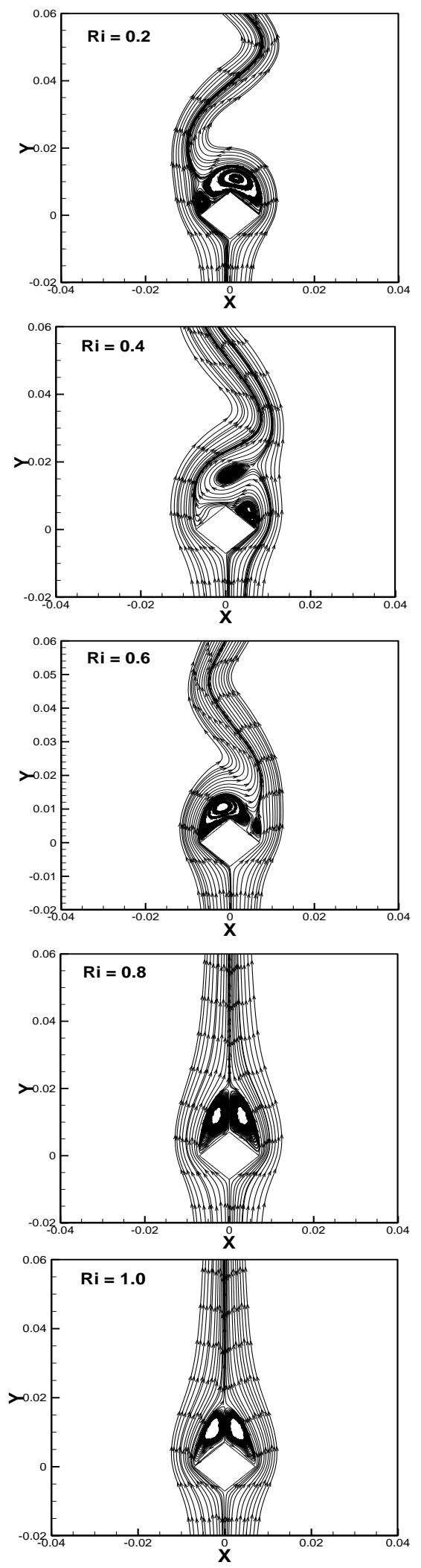

(a)
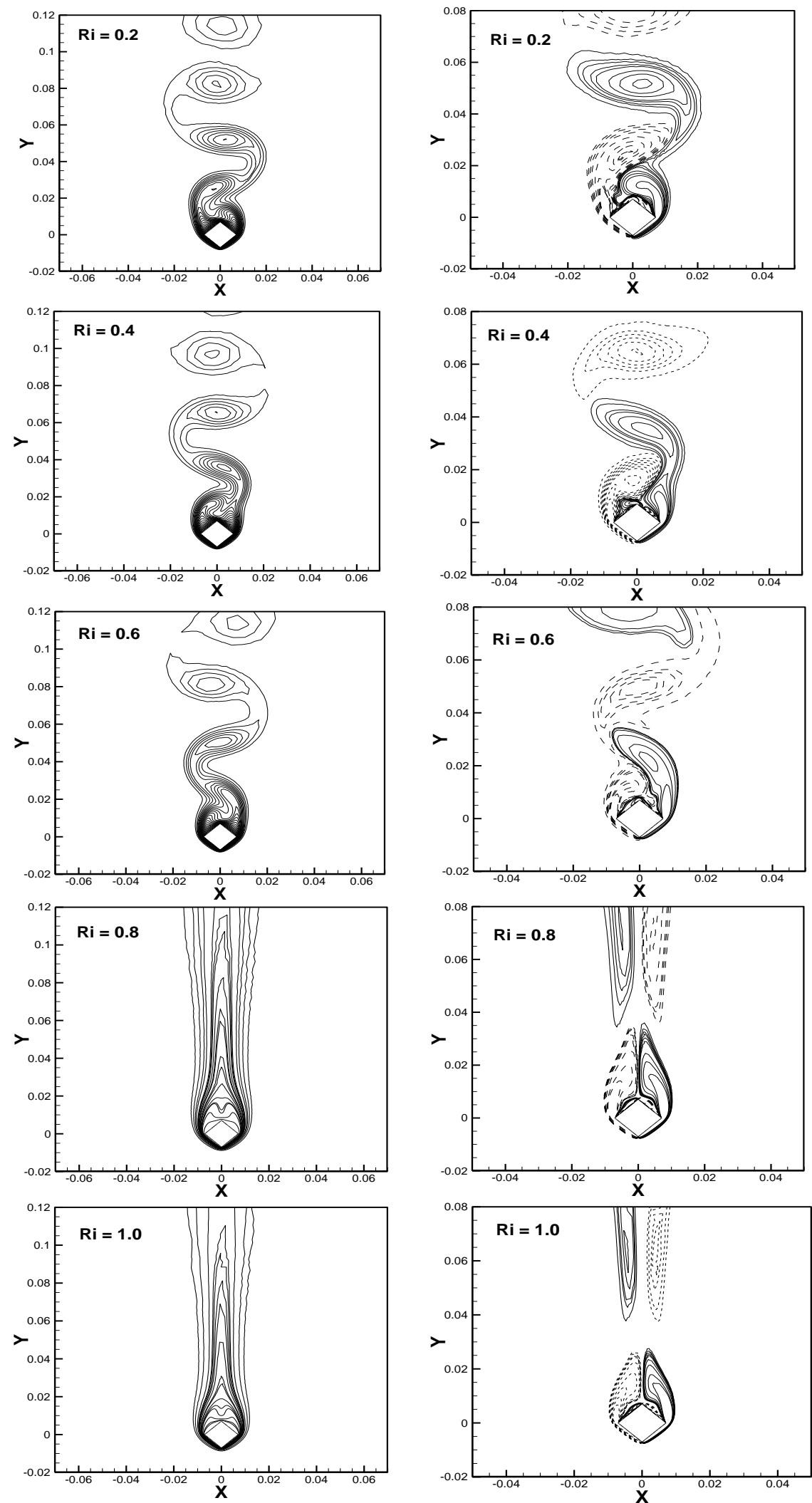

(b)

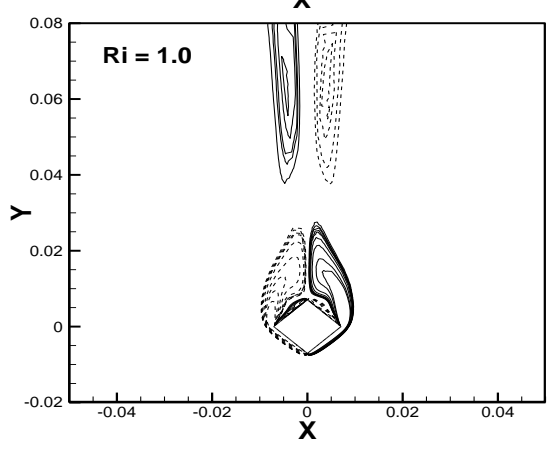

(c)

Figure 8. Different patterns at various Ri (a) Streamlines, (b) Isotherms, (c) Vorticity contours

\subsection{Effect of thermal buoyancy in aerodynamic and heat transfer parameters}

The characteristics of aerodynamic parameters (lift and drag coefficients) with $\mathrm{Ri}$ are also presented in the mixed convection regime. The Richardson number is varied as $0.2 \leq$ $\mathrm{Ri} \leq 1.0$ with an interval of 0.2 . The variations of $\mathrm{C}_{\mathrm{L}}$ and $\mathrm{C}_{\mathrm{D}}$ with $\mathrm{Ri}$ are shown in Figure 5. It is found that the mean lift coefficient has a nearly constant value close to zero at all Richardson number. This is due to the fact that at cylinder inclination $\phi=45^{\circ}$, the mean flow passes symmetrically on two equal halves of the cylinder. The mean coefficient of drag increases with an increase in Ri. At high Richardson number, the fluid accelerates to high velocities at the lower corner of 
the cylinder, resulting in much lower pressure. The pressure decreases significantly with an increase in $\mathrm{Ri}$ on the faces of a square cylinder located in the downstream region. This results in the increase of drag force and hence an increase in the mean drag coefficient.

The heat transfer parameter is the Nusselt number $(\mathrm{Nu})$, which is an important parameter to study. It represents the transfer of heat from the surface of the cylinder to the surrounding fluids. The effect of thermal buoyancy on the $\mathrm{Nu}$ is studied by performing numerical simulations at five different Richardson number i.e. $\mathrm{Ri}=0.2,0.4,0.6,0.8$ and 1.0. Figure 6 depicts the variation of mean $\mathrm{Nu}$ with $\mathrm{Ri}$. It is found that when the Richardson number is increased, the mean $\mathrm{Nu}$ also increases. The pattern of mean Nusselt number and mean coefficient of drag are similar. With an increase in Richardson number, buoyancy increases and hence heat transfer rate from the surface of the cylinder to surrounding fluids increases. This results in an increase of mean $\mathrm{Nu}$ with the increase in Richardson number.

The VS frequency in dimensionless form is called the Strouhal number. It is an important parameter for unsteady periodic flow. Figure 7 shows the variation of the Strouhal number with the Richardson number. It is found that as Richardson number is increased, Strouhal number increases and ultimately it vanishes at $\mathrm{Ri}=0.78$ where VS is suppressed. This is due to the fact that with an increase in $\mathrm{Ri}$, the shearlayer speeds-up resulting in an increase of circulation build up rate of the vortices. Thus, the shedding frequency of the vortices increases with the increase in $\mathrm{Ri}$, and when the buoyancy effect crosses the critical state, the suppression of vortices occurs, which results in zero shedding frequency and the unsteady flow becomes steady.

\subsection{Effect of thermal buoyancy on flow patterns}

The topology of a wake for the fluid flow past an inclined square cylinder can be studied by various flow patterns such as streamlines, isotherms, and vorticity contours. The instantaneous streamlines, isotherms and vorticity contours near the square cylinder at different Richardson numbers are shown in Figure 7. The effect of thermal buoyancy is studied for $\mathrm{Ri}$ varying as $0.2 \leq \mathrm{Ri} \leq 1.0$. As Richardson number increases, the velocity of the fluid in the wake increases thus weakening the shear layer and the roll-up process and hence the vortex shedding is suppressed. As already discussed in the preceding section, that suppression of vortex shedding occurs at $\mathrm{Ri}_{\mathrm{c}}=0.78$. It is found that when fluid flows past a square cylinder inclined at an angle of $45^{\circ}$, the separation point is fixed at the middle two corners of the cylinder. Thus, it seems that the most dominating factor for vortex shedding suppression is the acceleration of fluid velocity in the wake due to thermal buoyancy. Figure 8 shows that two stationary vortices are formed in the downstream region at $\mathrm{Ri}=0.8$ and $\mathrm{Ri}=1.0$, where there is a suppression of VS. It is found that there is a decrease in the size of downstream twin stationary vortices with increasing Richardson number. This was also mentioned by Sharma and Eswaran [2] for upward flow around a square cylinder in mixed convection regime at $\phi=0^{\circ}$, $\operatorname{Pr}=0.71$ and $\operatorname{Re}=100$.

\section{CONCLUSIONS}

The two-dimensional numerical investigations are done to study the influence of thermal buoyancy on the upward flow of air past a square cylinder inclined at an angle of $\phi=45^{\circ}$ for a fixed Reynolds number $(\operatorname{Re}=100)$. For this type of flows, a vortex shedding phenomenon occurs in the absence of any induced thermal buoyancy. The suppression of VS is done by imposing thermal buoyancy through the Richardson number. The critical $\mathrm{Ri}$ at which the VS is completely suppressed is determined by performing a series of numerical simulations near the bifurcation point. For the present flow conditions, it is concluded that the VS suppression occurs at $\mathrm{Ri}_{\mathrm{c}}=0.78$. The effect of thermal buoyancy on the aerodynamic parameters $\left(\mathrm{C}_{\mathrm{L}}\right.$ and $\mathrm{C}_{\mathrm{D}}$ ), heat transfer parameter $(\mathrm{Nu})$ and Strouhal number (St) are studied under the same flow conditions.

It is also concluded that the coefficient of drag increases with an increase in $\mathrm{Ri}$ and no lift is developed for any $\mathrm{Ri}$. The Nusselt number increases with Ri. While there is an increase in Strouhal number as $\mathrm{Ri}$ increases, till critical $\mathrm{Ri}$, and then it vanishes at $\mathrm{Ri}=0.78$. Further, the effect of thermal buoyancy on various flow patterns such as streamline, iso-vorticity contours and isotherms contours is studied. It is found that there is a decrease in the size of downstream twin stationary vortices with increasing Richardson number.

\section{ACKNOWLEDGMENT}

Authors acknowlege TEQIP-II and TEQIP-III for providing funds in establishing computing facilities in Computational Fluid Dynamics and High Performance Computing Laboratories, Zakir Husain College of Engineering \& Technology, Department of Mechanical Engineering, Aligarh Muslim University, Aligarh, India.

\section{REFERENCES}

[1] Ortega-Casanova, J. (2017). On the onset of vortex shedding from 2D confined rectangular cylinders having different aspect ratios: Application to promote mixing fluids. Chemical Engineering and Processing - Process Intensification, 120: 81-92. https://doi.org/10.1016/j.cep.2017.06.014

[2] Alfieri, F., Tiwari, M.K., Renfer, A., Brunschwiler, T., Michel, B., Poulikakos, D. (2013). Computational modeling of vortex shedding in water cooling of $3 \mathrm{D}$ integrated electronics. International Journal of Heat and Fluid Flow, 44: 745-755. https://doi.org/10.1016/j.ijheatfluidflow.2013.10.004

[3] Bhattacharyya, S., Singh, A.K. (2010). Vortex shedding and heat transfer dependence on effective Reynolds number for mixed convection around a cylinder in cross flow. International Journal of Heat and Mass Transfer, 53(15-16): 3202-3212. https://doi.org/10.1016/j.ijheatmasstransfer.2010.03.006

[4] Karthikeyan, S., Senthilkumar, S., Kannan, B.T., Chandrasekhar, U. (2018). Numerical analysis on effect of jet injection on vortex shedding for flow over a circular cylinder. Arabian Journal for Science and Engineering, 44: https://doi.org/10.1007/s13369-018-3588-1

[5] Rashid, A., Hasan, N. (2011). Vortex-shedding suppression in mixed convective flow past a heated square cylinder. World Academy of Science, Engineering and Technology, 54: 62-70. 
waset.org/publications/7771

[6] Khan, M.A., Anwer, S.F., Khan, S.A., Arif, M.R., Masood, S. (2019). Effect of thermal buoyancy on vortex induced vibration of square cylinder. In Proc. of 64th International Congress of the Indian Society of Theoretical and Applied Mechanics (ISTAM-2019), IIT Bhubaneswar, India held in December. 9-12.

[7] Chatterjee, D., Sinha, C. (2014). Influence of thermal buoyancy on vortex shedding behind a rotating circular cylinder in cross flow at subcritical Reynolds numbers. Journal of Heat Transfer, 136(5): 051704 https://doi.org/10.1115/1.4026007

[8] Choi, H., Jeon, W., Kim, J. (2016). Control of flow over a bluff body. Annual Review of Fluid Mechanics, 40: 113-39.

https://doi.org/10.1146/annurev.fluid.39.050905.110149

[9] Rashidi, S., Hayatdavoodi, M., Esfahani, J.A. (2016). Vortex shedding suppression and wake control: A review. Ocean Engineering, 126: 57-80. https://doi.org/10.1016/j.oceaneng.2016.08.031

[10] Lecordier, J.C., Hamma, L., Paranthoen, P. (1991). The control of vortex shedding behind heated circular cylinders at low Reynolds numbers. Experiments in Fluids, 10(4): 224-229. https://doi.org/10.1007/BF00190392

[11] Lecordier, J.C., Browne, L.W.B., Le Masson, S., Dumouchel, F., Paranthoën, P. (2000). Control of vortex shedding by thermal effect at low Reynolds numbers. Experimental Thermal and Fluid Science, 21(4): 227-237. https://doi.org/10.1016/S0894-1777(00)00007-8

[12] Sarioglu, M. (2017). Control of flow around a square cylinder at incidence by using a splitter plate. Flow Measurement and Instrumentation, 53: 221229.https://doi.org/10.1016/j.flowmeasinst.2016.06.024

[13] Salimipour, E. (2019). A numerical study on the fluid flow and heat transfer from a horizontal circular cylinder under mixed convection. International Journal of Heat and Mass Transfer, 131: 365-374. https://doi.org/10.1016/j.ijheatmasstransfer.2018.11.084

[14] Arif, M.R., Hasan, N. (2019). Performance of characteristic numerical boundary conditions for mixed convective flows past a heated square cylinder using a non-Boussinesq approach. Numerical Heat Transfer, Part A: Applications, 76(4): 254-280. https://doi.org/10.1080/10407782.2019.1627828

[15] Singh, S., Chandar, D. (2016). Effects of thermal induced buoyancy forces on the vortex shedding of a circular cylinder. International Communications in Heat and Mass Transfer, 76: 215-224. https://doi.org/10.1016/j.icheatmasstransfer.2016.05.01 2

[16] Arif, M.R., Hasan, N. (2019). Effect of cylinder inclination and heating on mixed convective flows past a square cylinder using non-Boussinesq model. Proc Indian Soc Theor Appl Sci.

[17] Sharma, A., Eswaran, V. (2004). Effect of aiding and opposing buoyancy on the heat and fluid flow across a square cylinder at $\mathrm{Re}=100$. Numerical Heat Transfer, Part A: Applications, 45(6): 601-624. https://doi.org/10.1080/10407780490277798

[18] Bhattacharyya, S., Mahapatra, S. (2005). Vortex shedding around a heated square cylinder under the influence of buoyancy. Heat and Mass Transfer, 41(9): 824-833. https://doi.org/10.1007/s00231-005-0626-9
[19] Hasan, N., Ali, R. (2013). Vortex-shedding suppression in two-dimensional mixed convective flows past circular and square cylinders. Physics of Fluids, 25(5): 88. https://doi.org/10.1063/1.4804387

[20] Hasan, N., Saeed, A. (2017). Effects of heating and freestream orientation in two-dimensional forced convective flow of air past a square cylinder. International Journal of Thermal Sciences, 112: 1-30. https://doi.org/10.1016/j.ijthermalsci.2016.08.020

[21] Chatterjee, D., Mondal, B. (2012). Effect of thermal buoyancy on the two-dimensional upward flow and heat transfer around a square cylinder. Heat Transfer Engineering, $\quad 33(12)$ : https://doi.org/10.1080/01457632.2012.659634

[22] Sahu, A.K., Chhabra, R.P., Eswaran, V. (2009). Effects of Reynolds and Prandtl numbers on heat transfer from a square cylinder in the unsteady flow regime. International Journal of Heat and Mass Transfer, 52(3-4): 839-850. https://doi.org/10.1016/j.ijheatmasstransfer.2008.07.032

[23] Tanweer, S., Dewan, A., Sanghi, S. (2019). Study on effects of Prandtl number on cross buoyancy flow past a square cylinder using OpenFOAM. Journal of Applied Fluid Mechanics, 12(1): 257-269. https://doi.org/10.18869/acadpub.jafm.75.253.29020

[24] Elsherbiny, S.M., Teamah, M.A., Moussa, A.R. (2017). Experimental mixed convection heat transfer from an isothermal horizontal square cylinder. Experimental Thermal and Fluid Science, 82: 459-471. https://doi.org/10.1016/j.expthermflusci.2016.12.002

[25] Yoon, D.H., Yang, K.S., Choi, C.B. (2010). Flow past a square cylinder with an angle of incidence. Physics of Fluids, 22(4):

043603. https://doi.org/10.1063/1.3388857

[26] Sohankar, A., Norberg, C., Davidson, L. (1998). LowReynolds-number flow around a square cylinder at incidence: study of blockage, onset of vortex shedding and outlet boundary condition. International Journal for Numerical Methods in Fluids, 26(1): 39-56. https://doi.org/10.1002/(SICI)10970363(19980115)26:1<39::AID-FLD623>3.0.CO;2-P

[27] Dutta, S., Panigrahi, P.K., Muralidhar, K. (2004). Effect of orientation on the wake of a square cylinder at low Reynolds numbers. Indian Journal of Engineering and Materials $\quad$ Sciences, 11(6): 447-59. http://hdl.handle.net/123456789/9325

[28] Ranjan, R., Dalal, A., Biswas, G. (2008). A numerical study of fluid flow and heat transfer around a square cylinder at incidence using unstructured grids. Numerical Heat Transfer, Part A: Applications, 54(9): 890-913. https://doi.org/10.1080/10407780802424361

[29] Dulhani, J.P., Sarkar, S., Dalal, A. (2014). Effect of angle of incidence on mixed convective wake dynamics and heat transfer past a square cylinder in cross flow at $\mathrm{Re}=$ 100. International Journal of Heat and Mass Transfer, 74: 319-332.

https://doi.org/10.1016/j.ijheatmasstransfer.2014.03.021

[30] Kakade, A.A., Singh, S.K., Panigrahi, P.K., Muralidhar, K. (2010). Schlieren investigation of the square cylinder wake: Joint Influence of Buoyancy and Orientation. Physics of Fluids, 22(5): 054107. https://doi.org/10.1063/1.3415228

[31] Arif, M.R., Hasan, N. (2019). Vortex shedding suppression in mixed convective flow past a square 
cylinder subjected to large-scale heating using a nonBoussinesq model. Physics of Fluids, 31(2): 023602. https://doi.org/10.1063/1.5079516

[32] Sharma, A., Eswaran, V. (2005). Effect of channelconfinement and aiding/opposing buoyancy on the twodimensional laminar flow and heat transfer across a square cylinder. International Journal of Heat and Mass Transfer, 48(25-26): 5310-5322. https://doi.org/10.1016/j.ijheatmasstransfer.2005.07.027

[33] Yang, G., Wu, J. (2013). Effect of side ratio and aiding/opposing buoyancy on the aerodynamic and heat transfer characteristics around a rectangular cylinder at low Reynolds numbers. Numerical Heat Transfer, Part A: Applications, $\quad$ 64(12): 1016-1037. https://doi.org/10.1080/10407782.2013.811057

[34] Ansys, I. (2011). ANSYS FLUENT Theory Guide. Canonsburg, $\mathrm{Pa}, 794$.

[35] Chatterjee, D., Mondal, B. (2014). Control of flow separation around bluff obstacles by superimposed thermal buoyancy. International Journal of Heat and Mass Transfer, 72: 128-138. https://doi.org/10.1016/j.ijheatmasstransfer.2014.01.013

\section{NOMENCLATURE}

$\begin{array}{ll}\mathrm{Re} & \text { Reynolds number } \\ \mathrm{Pr} & \text { Prandtl number } \\ \mathrm{Ri} & \text { Richardson number } \\ \mathrm{St} & \text { Strouhal number } \\ \mathrm{Nu} & \text { Nusselt number } \\ \mathrm{f} & \text { dimensional VS frequency } \\ \mathrm{C}_{\mathrm{L}} & \text { coefficient of lift } \\ \mathrm{C}_{\mathrm{D}} & \text { coefficient of drag }\end{array}$

$\begin{array}{ll}\mathrm{F}_{\mathrm{L}} & \text { lift force } \\ \mathrm{F}_{\mathrm{D}} & \text { drag force } \\ \mathrm{Q} & \text { total heat transfer rate per unit cylinder span } \\ \mathrm{k} & \text { fluids thermal conductivity } \\ \mathrm{d} & \text { square cylinder edge } \\ \mathrm{U}_{\infty} & \text { free-stream velocity } \\ \mathrm{T} & \text { dimensionless temperature } \\ \mathrm{X}, \mathrm{y} & \text { dimensional cartesian coordinates } \\ \mathrm{X}, \mathrm{Y} & \text { non-dimensional cartesian coordinates } \\ \mathrm{u}, \mathrm{v} & \text { dimensional } \mathrm{x} \text { and y components of velocity } \\ \mathrm{U}, \mathrm{V} & \text { dimensionless } \mathrm{x} \text { and y components of } \\ \mathrm{p} & \text { velocity } \\ \mathrm{P} & \text { dimensional pressure } \\ \mathrm{t} & \text { non-dimensional pressure }\end{array}$

\section{Greek symbols}

$\phi$

$\theta$

$\tau$

$\rho$

$\mu$

$\beta$

$\varepsilon$

$\checkmark$

$\alpha$

\section{Subscripts}

$\begin{array}{ll}\infty & \text { free-stream conditions } \\ \mathrm{W} & \text { cylinder wall conditions } \\ \mathrm{c} & \text { critical value } \\ \mathrm{o} & \text { reference conditions }\end{array}$

cylinder inclination non-dimensional temperature non-dimensional time non-dimensional density of the fluid fluid viscosity volumetric expansion coefficient heating level fluids kinematic viscosity thermal diffusivity of fluids

reference conditions 